\title{
Esthetic \& Function: The 4D Solution A Clinical Evaluation
}

\section{Sebastien Felenc* and Maxime Jaisson}

Department of Prosthodontics, University of Montpellier, France

Received: 眥April 19, 2018; Published: 制 May 15, 2018

*Corresponding author: Sebastien Felenc, Ex-assistant professor, Department of Prosthodontics, University of Montpellier, France

\section{Background}

Since the beginning of dentistry, prosthodontists are trying to understand jaw motion and its impact on occlusion. This medical approach is dedicated firstly to achieve a diagnosis and then to be able to produce dental prosthesis with fully bespoke anatomies for each patient. Scientific literature is rich of technics invented to record jaw motion, especially between 1960 to 1990. Those devices were globally time consuming and required deep familiarity with occlusion making its use reserved for specialists [1-3].

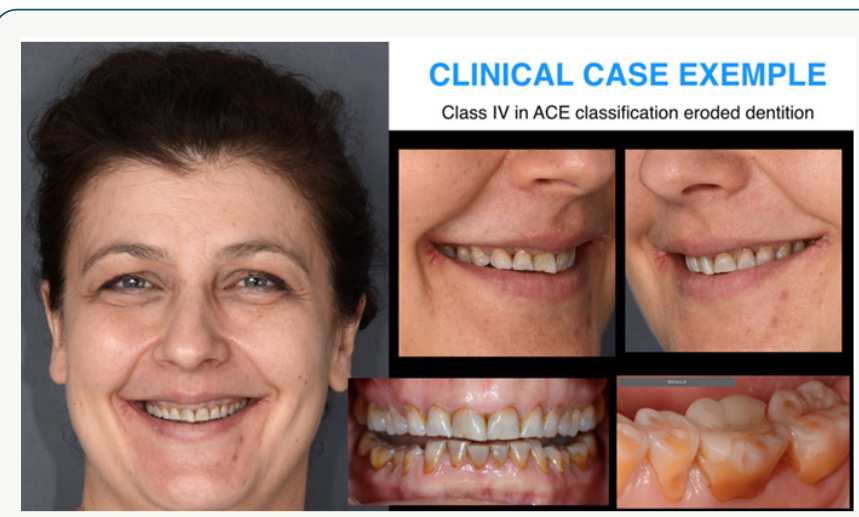

Picture 1: Initial situation of the clinical exemple. The patient presents muscular pain, cheilosis and hypersensitivity on the teeth. She shows a Class IV ACE classification for eroded dentitions.

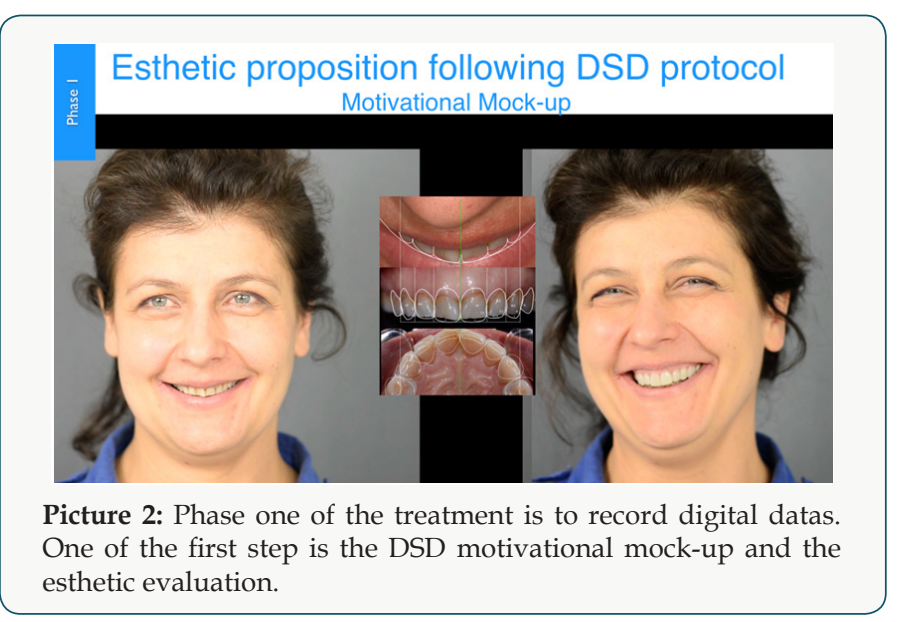

Clinicians and researchers filled the science with great knowledge about chewing patterns, condylar movements and jaw motion. But they were limited by technology when it came to use those informations for prosthetics production. Articulators reproduces jaw movements with a mechanical behavior, they can be programmed in order to increase the precision, but again, this action is time consuming and rather elitist (Pictures 1-5).
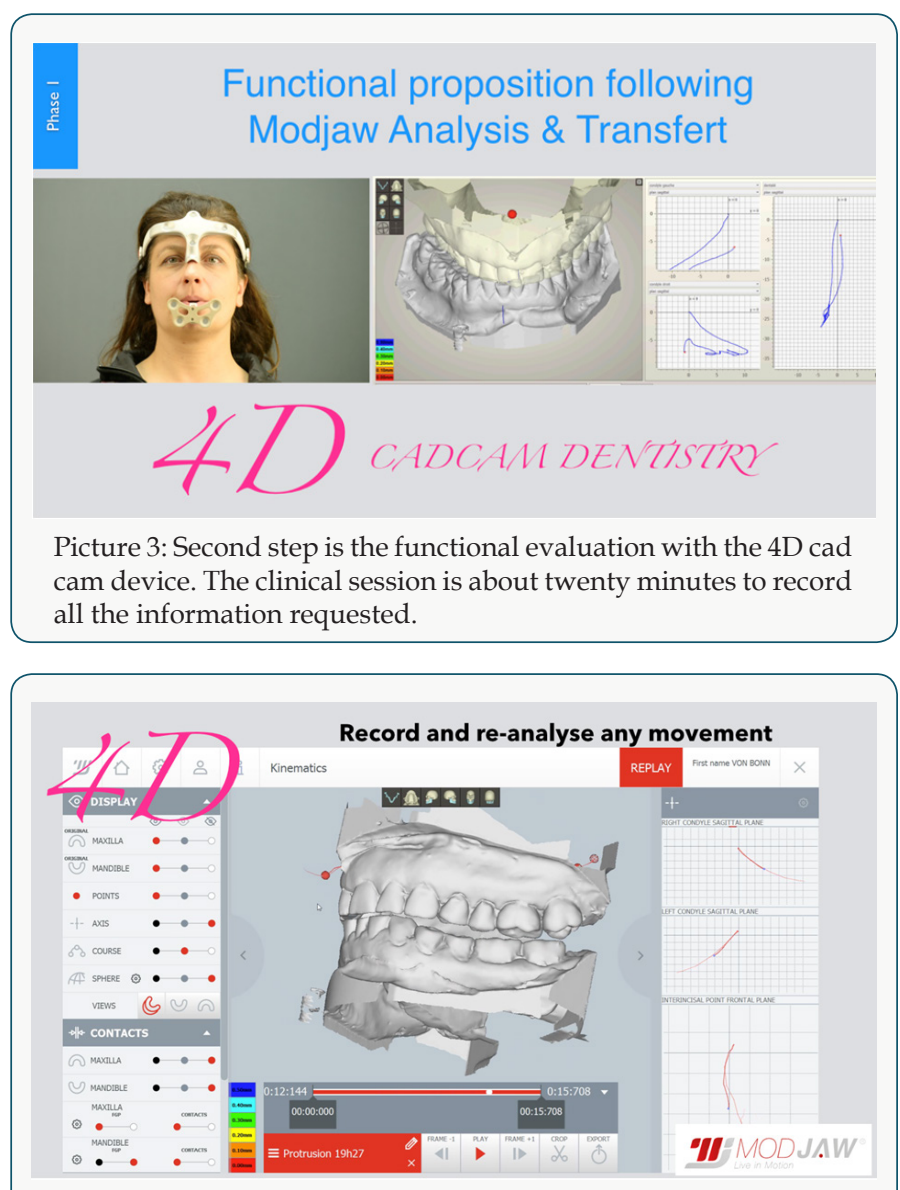

Picture 4: During the recording the evaluation is live so the clinician can ask and check any patient movement and contact. Then, all those informations can be re-played for further analysis. 


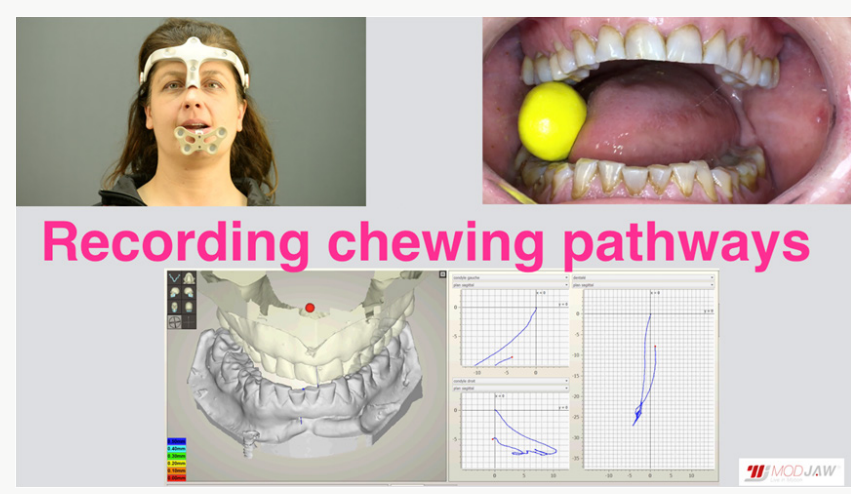

Picture 5: Recording real chewing pathways is the main revolution due to this $4 \mathrm{D}$ new technology. And more than just the analysis we will be able to penetrate the digital workflow with those informations.

For a few decades now, Cadcam dentistry resulted in the ability to build prosthetics using 3D virtual models and which are then milled or printed. But jaw motion was still an approximation and do not reflect the true patient's jaw movements. This will remain as long as virtual articulators stays digital copycats of mechanical ones bearing the same operating logics.

The aim of this presentation is to explain and evaluate a new approach based on a digital record of the jaw motion: 3D models are animated just as the patient's function replicating the exact same jaw movement over time, this is why it is called the 4D Cadcam technology. To be long lasting, our prosthetic restorations must be functionally integrated, esthetic and perfectly correlated with the patient's function.

\section{Material \& Methods}

The innovative approach lies in the combination of 3D technologies and motion capture. A tracking device with an optical camera follows the displacement of patient's morphological points in space with a very high frequency and precision. A set of trackers are placed on the patient's head and in the patient's mouth. They are correlated to pre-recorded 3D models thanks to a specific hand piece. The optical camera uses infrared for the tracking and transfers data to a dedicated software. When the jaw is in movement, the tracking camera records the point animations, and in the same time the software makes 3D models reproduces the motion. Computational geometry enables the 3D software to show the contact points and the dynamic mapping of teeth contacts. All the jaw movements are in real time and recorded. This allows the clinician to directly analyse, replay and re-do any movement if necessary, and re-interpret the recordings. The models can be observed with the ease of $3 \mathrm{D}$ appliance and through the time recording of the jaw motion, this is why it is called 4D CadCam technologies [4-6].

5 years of research and development allowed the actual version of this device to present a high level of repeatable precision and to be presented to the public as a prototype. The tracking camera precision is between $5 \mathrm{um}$ and $70 \mathrm{um}$ with a recording frequency of $120 \mathrm{Htz}$. Test bench measurements showed a precision between reality and software from $50 \mathrm{um}$ to $90 \mathrm{um}$. Scientific studies for the evaluation of the precision of mapping contacts and condyle displacement are currently carried out by Montpellier II Université (France). Since 2013 the device has been clinically evaluated on 54 cases. Each case was documented with photos and videos, each patient was recorded thru the Modjaw solution and digital workflows were created (Pictures 6-10).
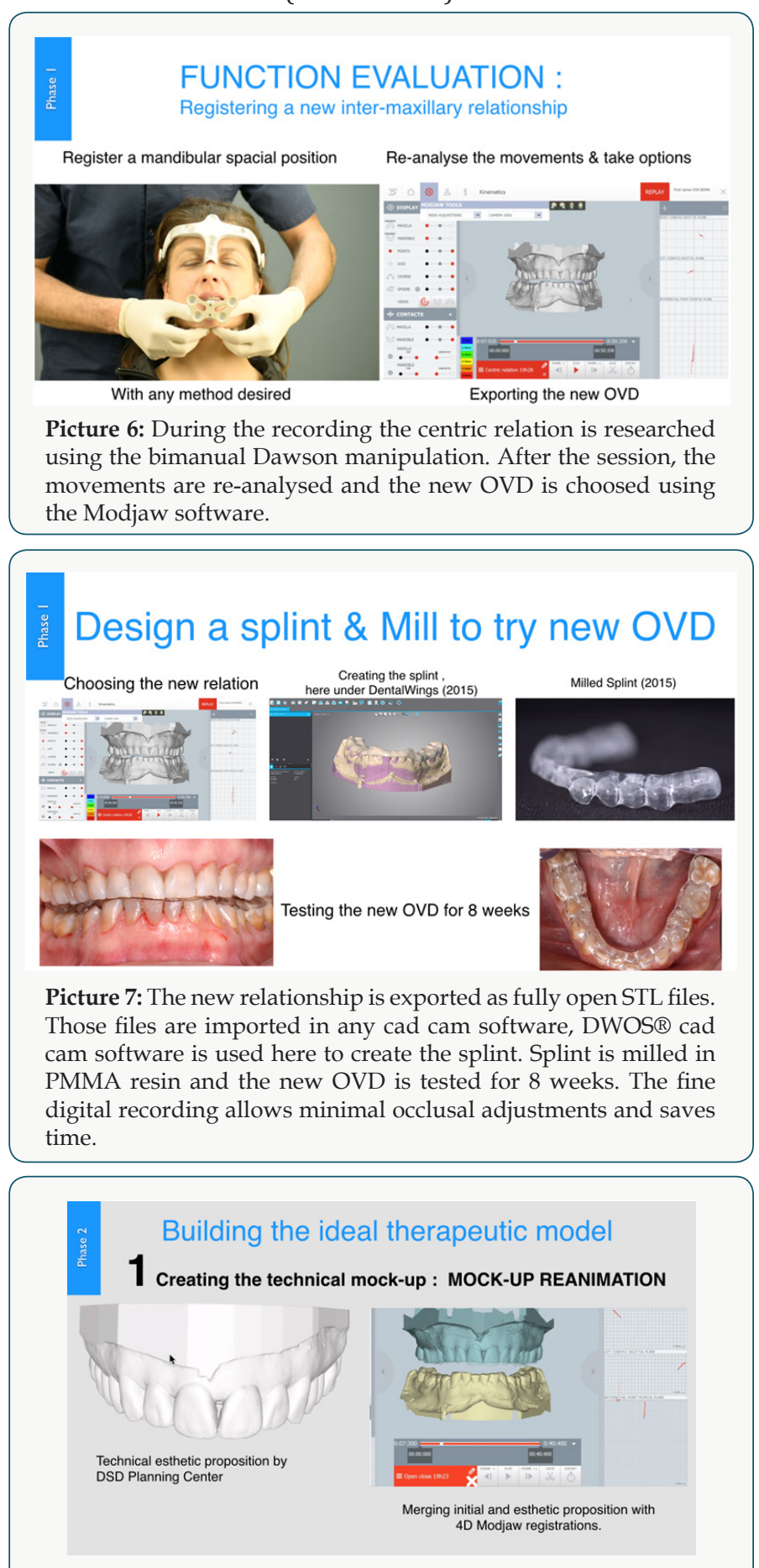

Picture 8: As the new OVD is confirmed we can now build the ideal therapeutic model, merging informations from aesthetic and function : this is the second step. Modjaw software will reanimate the digital proposition with patient's real function in order to observe the function impact on the aesthetic outcome. 


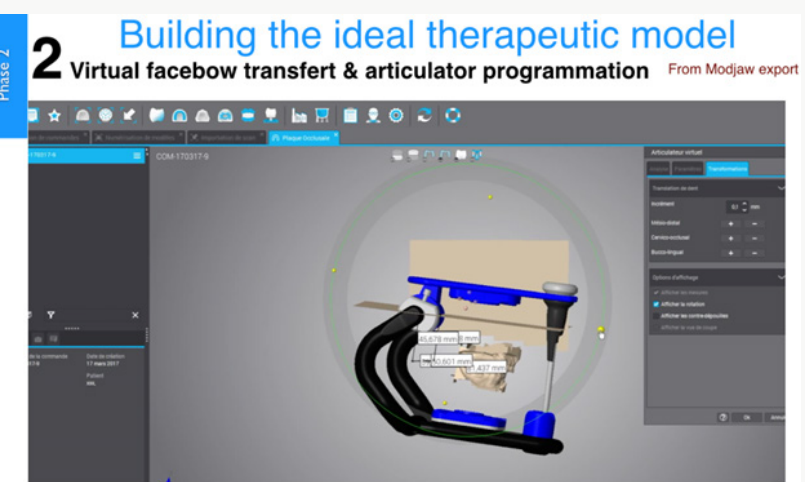

Picture 9: One of the basic functionality of the Modjaw device is to record and export the maxillary spatial position. This is the digital face bow function. This step is mandatory in order to use digital articulators.

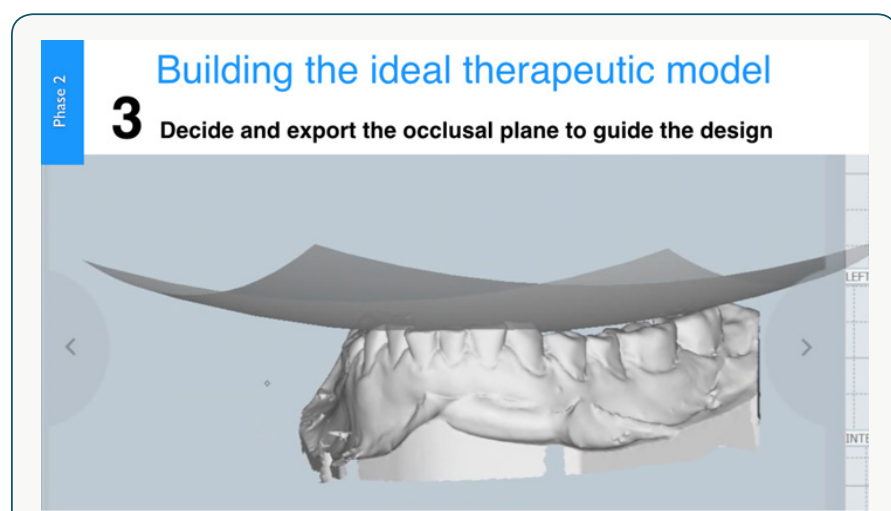

Picture 10: Another functionality is to pre-position an occlusal plane according to Monson's sphere. This setting done by the clinician is exported with the STL files in order to guide the digital design of the posteriors.

\section{3 sections were evaluated:}

a. Clinical relevance as a digital axiograph, performed in pre, per and post-operative treatment.

b. Digital workflows thru Modjaw exports, datas implementation to cadcam laboratory softwares leading to the production of dental prosthesis.

c. Clinical and laboratory relevance in terms of time saving and precision delivering.

13 cases were functionally evaluated; leading to occlusal diagnosis, research for the development of the solution and without any dental treatment. 21 registered cases were used to diagnosis and occlusal therapy was performed as equilibration and eventually splint. 25 registered cases were used to build dental prosthesis of any kind, from complete dentures, to fixed and bonded restaurations.

\section{Results \& Conclusions}

The 3 decisive characteristics of this device are

a. Ergonomic and ease to use thanks to new digital technologies, meaning that 15 minutes are sufficient to record perfectly patient's jaw motion.

b. None invasive and very light set up especially in the mouth which is key not to create bias in the motion analysis.

c. The relevance of the recorded information and the ease of its use directly in the current digital workflow.

Through the example of a clinical case we will present the range of possibilities offered by the device leading to create new pathways in aesthetic and functional prosthetic therapeutics (Pictures 1114).

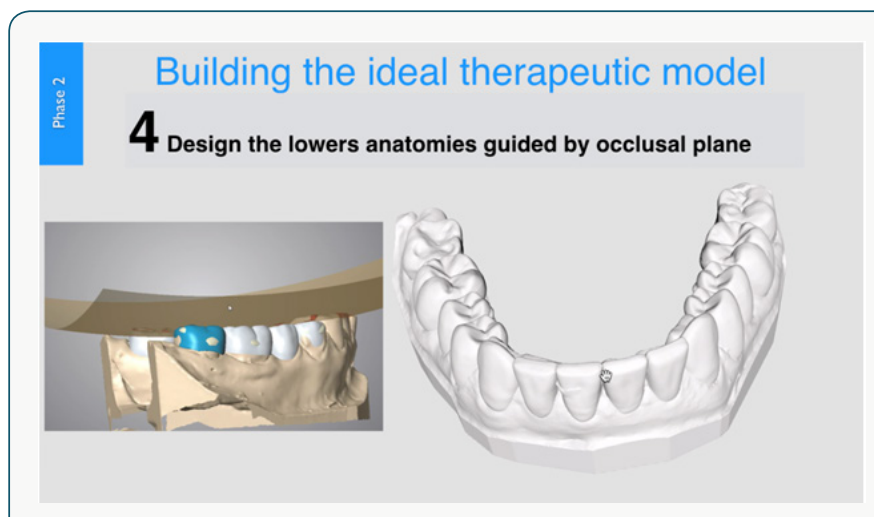

Picture 11: Lower anatomies are designed facing the occlusal exported plane.
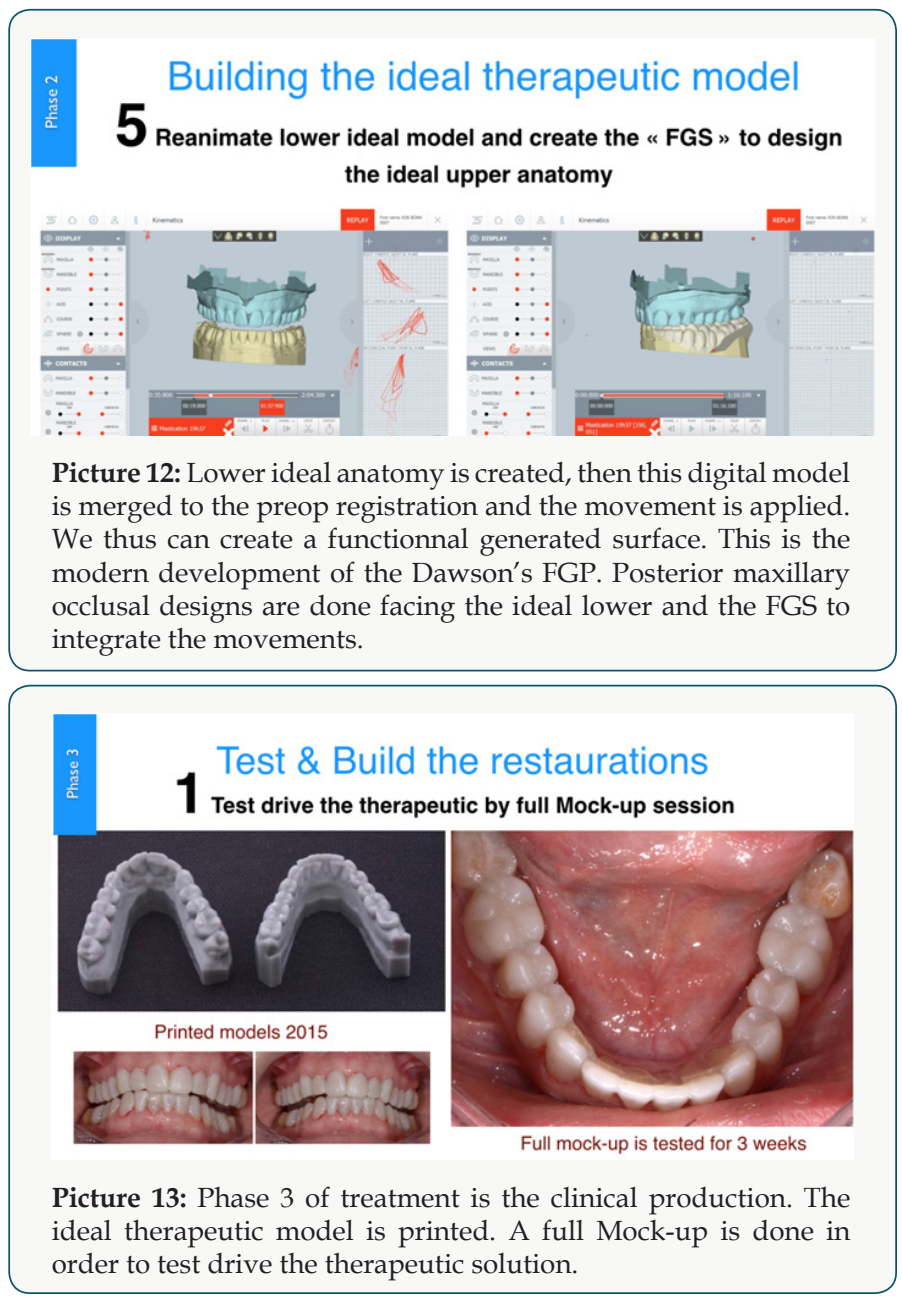


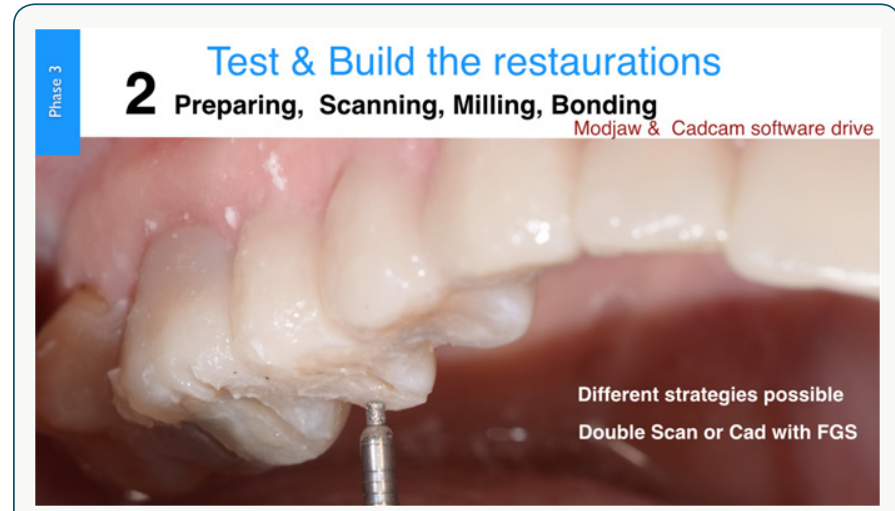

Picture 14: After 3 weeks of evaluation minimally invasive restorations are prepared directly thru the mock-up. We use here special drills for assisted guided preparations.

The global strategy is to pre-record a maximum of digital informations, work the case upstream to minimize the patient's time on the chair. The clinical case is chronologically followed in order to have a full overview of the workflow. Within this case study we will perfom a pre-operative functional and aesthetic analysis, raise the vertical dimension in occlusion, choose the new inter maxilla relationship, virtually position the occlusal plane, create a deduceted FGP (Functional Generaled Path), transfer everything thru a digital face-bow to a digital articulator in order to create the best digital therapeutic model possible. Then this therapeutic solution is tested in reality with full oral mock-ups for validation. Finally the prosthetic treatment is performed. This new solution gives us more elements for in-depth diagnosis in a very short time and without any specific skill required. It streamlines the digital workflow while opening new opportunities with current CADCAM technologies. It provides quickly the laboratory with a key set of data directly usable to create prosthetics, allowing the clinician and the technician to save time compared to the usual workflow.

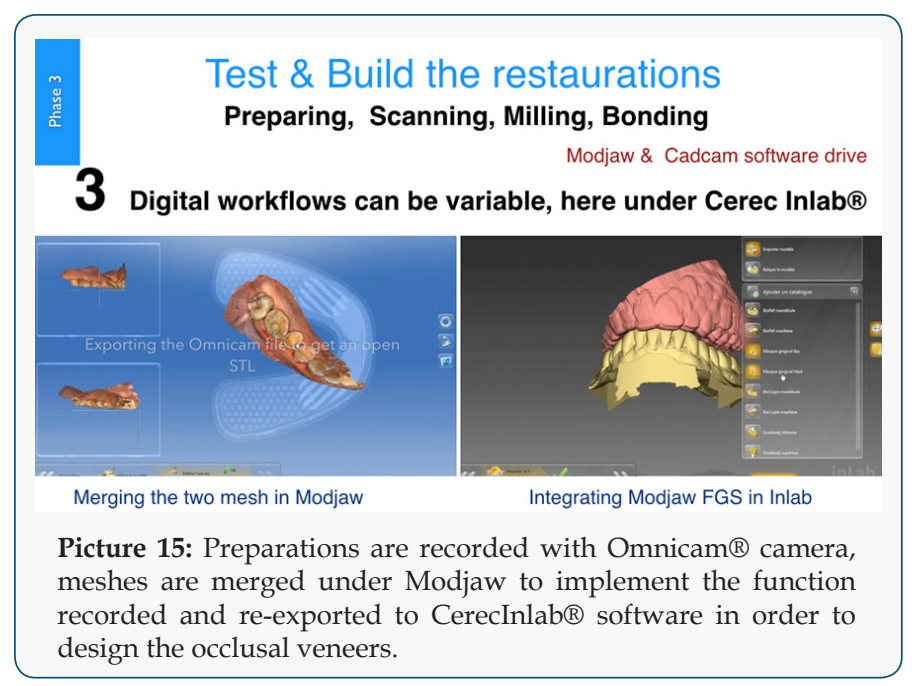

At last but not least it allows the team to build individually driven occlusions, with less patient adaptation to the prothesis and certainly better long-lasting aesthetic restorations. A comparaison can be done with the evolution from maps to GPS (global positioning system) in navigation, leading to more information, less approximations and great evolution. This technology strengthens our therapy freedom, and as usual with freedom it requires more of us because we have more options, more to decide (so many possibilities) and to take action [7-9] (Pictures 15-17).

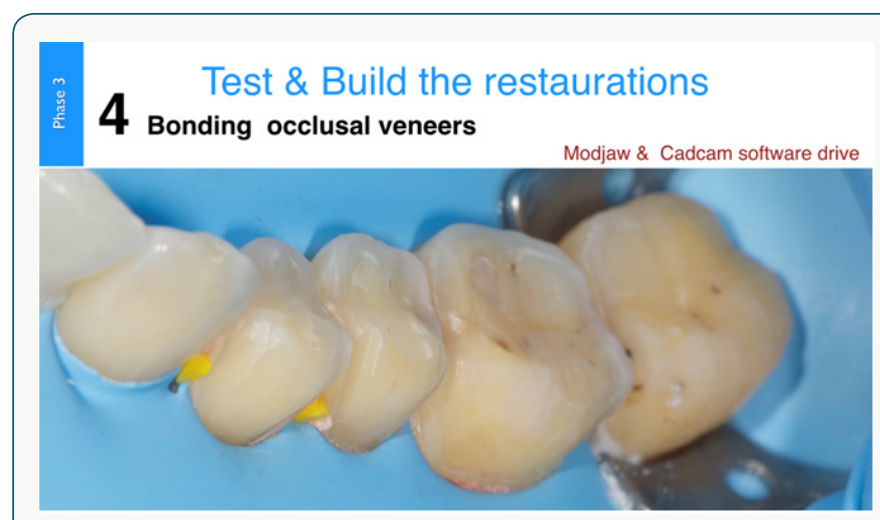

Picture 16: Ending phase 3 of treatment : local isolation before bonding. This is mainstream.

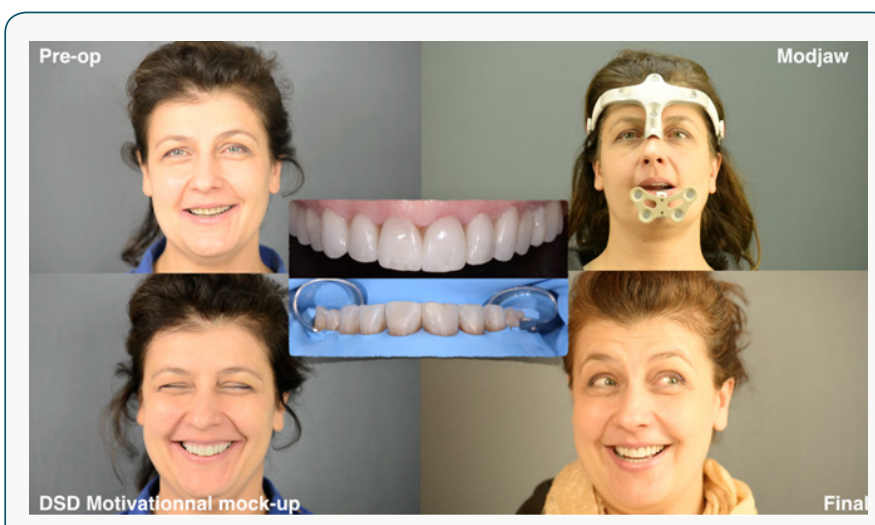

Picture 17: General resume of the digital workflow. The patient is recorded with, 2D, 3D and now $4 \mathrm{D}$ technology. This approach streamlines the treatment and minimizes the patient's time on the chair.

\section{References}

1. (2008) Dental conclusion: a critical reflexion on past, present and futur concepts. J oral Rehab 35(6): 446-453.

2. Jemt, Karlson (1982) Computer analysed movements in three dimensions recorded by light emitting diodes. J Oral Rehab 9317-326.

3. HOBO S, coll (1982) Study of mandibular movements by means of electronic measuring system. J Japan Prostch Soc 26(3) : 619-634.

4. Lundeen HC, Shryock EF, Gibbs CH (1978) An evaluation of mandibular border movements: their character and significance. J Prosthet Dent 40(4): 442-452.

5. Solaberrieta E, Garmendia A, Minguez R, Brizuela A, Pradies G (2015) Virtual facebow technique J Prosthet Dent 114(6): 751-755.

6. Solaberrieta E, Minguez R, Otegi JR, Etxaniz O (2014) Improved digital transfer of the maxillary cast to a virtual articulator. J Prosthet Dent. 112(4): 921-924.

7. Solaberrieta E, Minguez R, Barrenetxea L, Etxaniz O (2013) Direct transfer of the position of digitized casts to a virtual articulator. J Prosthet Dent 109(6): 411-414. 
8. Lux LH, Thompson GA, Waliszewski KJ, Ziebert GJ (2015) Comparison of the Kois Dento-Facial Analyzer System with an earbow for mounting a maxillary cast. J Prosthet Dent 114(3): 432-439.
9. Laura H,Lux, Geoffrey A (2015) Thompson, Kenneth J. Waliszewski, Gerald J. Ziebert Journal of Prosthetic Dentistry 114(3): 432-439. (c) This work is licensed under Creative

To Submit Your Article Click Here: $\quad$ Submit Article

DOI: 10.32474/MADOHC.2018.02.000137

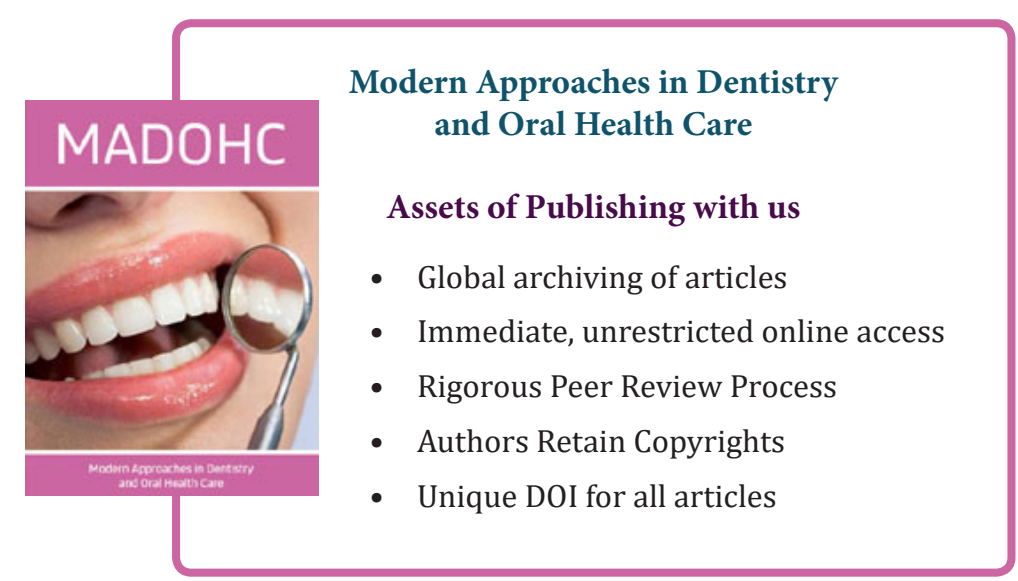

